\title{
artigo
}

Robson da Silva, R.; Souza, M.V.L.; Alencar, I.F.; Inácio, A.F.L.; Ferreira da Silva, D.; Messias, I.F.; Magalhães, A.F.L.;

Neuropatias diabéticas periféricas como complicações do diabetes mellitus: estudo de revisão

\section{Neuropatias diabéticas periféricas como complicações do diabetes mellitus: estudo de revisão}

\author{
Peripheral diabetic neuropathies as complications of diabetes mellitus: review study
}

Neuropatías diabéticas periféricas como complicaciones de la diabetes mellitus: estudio de revisión

\begin{abstract}
RESUMO
Objetivo: este estudo objetivou a evidenciar publicações expressas na literatura cientifica mundial sobre essa temática. Métodos: trata-se de uma revisão integrativa que seguiu a estratégia pico para identificar os fatores associados a neuropatia diabética. A busca dos artigos foi realizada em três bases de dados eletrônicas: medline, biblioteca virtual de saúde e scielo. Foram utilizados os descritores "neuropatias diabéticas", "neuralgia", "fatores de risco", "diabetes mellitus", com textos completos, publicados entre 2011 a 2021. Resultado: foram encontrados 118 artigos. Cinquenta e cinco artigos foram selecionados para serem lidos na íntegra e 5 atenderam aos critérios desta revisão. Conclusão: a neuropatia diabética pode ser reduzida com melhor controle da glicose no sangue, e a melhora dos índices de lipídios e pressão arterial.
\end{abstract}

DESCRITORES: neuropatias diabéticas; neuralgia; fatores de risco; diabetes mellitus.

\section{ABSTRACT}

Objective: this study aimed to highlight publications expressed in the worldwide scientific literature on this topic. Methods: this is an integrative review that follows a pico strategy to identify the factors associated with diabetic neuropathy. The search for articles carried out in three electronic databases: medline, virtual health library and scielo. The descriptors "diabetic neuropathies", "neuralgia", "risk factors", "diabetes mellitus" were used, with complete texts, published between 2011 and 2021. Result: 118 articles were found. Fifty-five articles were selected to be read in full and 5 met the criteria of this review. Conclusion: diabetic neuropathy can be reduced with better blood glucose control, and improved lipid and blood pressure levels.

DESCRIPTORS: diabetic neuropathies; neuralgia; risk factors; diabetes mellitus.

\section{RESUMEN}

Objetivo: este estudio tuvo como objetivo destacar las publicaciones en la literatura científica mundial sobre este tema. Métodos: se trata de una revisión integradora que siguió la estrategia pico para identificar los factores asociados a la neuropatía diabética. La búsqueda de los artículos se realizó en bases de datos electrónicas: medline, biblioteca virtual en salud y scielo. Se utilizaron los descriptores "neuropatías diabéticas", "neuralgia", "factores de riesgo", "diabetes mellitus", con textos completos, publicados entre 2011 y 2021. Resultado: se encontraron 118 artículos. Se seleccionaron cincuenta y cinco artículos para ser leídos en su totalidad y cinco cumplieron los criterios de esta revisión. Conclusión: la neuropatía diabética se puede reducir con un mejor control de la glucosa en sangre y mejores niveles de lípidos y presión arterial.

DESCRIPTORES: neuropatías diabéticas; neuralgia; factores de riesgo; diabetes mellitus.

RECEBIDO EM: 21/04/2021 APROVADO EM: 24/05/2021

\section{Roni Robson da Silva}

Acadêmico de Enfermagem pela Universidade Veiga de Almeida (UVA). Pós-graduando em MBA Gestão em Saúde pela Escola de Enfermagem da Universidade de São Paulo-EERP/USP.

ORCID: 0000-0001-6010-6438

\section{Marcus Vinicius Lessa de Souza}

Enfermeiro. Especialista em Terapia Intensiva e Geriatria e Gerontologia. Pós graduando em Cardiologia Intensiva de Alta Complexidade. Enfermeiro Rotina CTI - Rede D'or São Luiz.

ORCID: 0000-0002-3023-4778 


\section{Icaro Ferracini Alencar}

Acadêmico de Medicina pela Universidade Iguaçu UNIG

ORCID: 0000-0003-3852-7767

\section{Andréa Ferreira Leite Inácio}

Enfermeira. Especialista em Urgência e Emergência, Comissão de Controle de Infecção Hospitalar CCIH, Especialista em Docência do Ensino Superior e Estratégia Saúde da Família ESF.

ORCID: 0000-0002-7132-4892

\section{Diego Ferreira da Silva}

Enfermeiro Assistencial na UTI Adulto do Hospital Universitário da Universidade Federal de São Paulo UNIFESP. Mestrando Escola de Enfermagem da Universidade de São Paulo-EE/USP no programa PROESA. Especialista modalidade Residência Multiprofissional em Neurologia e Neurocirurgia pela Universidade Federal de São Paulo UNIFESP.

ORCID: 0000-0001-8275-7178

\section{Icaro Ferro Messias}

Médico. Graduado pela Universidade Federal do Rio Grande do Sul (UFRGS).

ORCID: 0000-0003-4632-1734

\section{André Felipe Lemos de Magalhães}

Enfermeiro. Pós Graduação Lato Sensu em Saúde Estética pela Faculdade de Tecnologia e Ciência do Alto Paranaíba: Paranaíba, Minas Gerais, BR

ORCID: 0000-0002-5554-0798

\section{INTRODUÇÃO}

0 diabetes mellitus tipo $1(\mathrm{dm} 1)$ é uma doença autoimune crônica, sendo na maioria dos casos diagnosticado em crianças e adolescentes, é uma doença causada pela produção insuficiente ou má absorção de insulina, hormônio que regula a glicose no sangue e garante energia para o organismo ${ }^{1}$. A insulina é um hormônio que tem a função de quebrar as moléculas de glicose transformando-a em energia para manutenção das células do nosso organismo ${ }^{2}$. O diabetes pode causar o aumento da glicemia e as altas taxas podem levar a complicações no coração, artérias, olhos, rins e nervos ${ }^{3}$. Em casos mais graves, o diabetes pode levar à morte. No brasil, de acordo com a sociedade brasileira de diabetes, existem atualmente, mais de 13 milhões de pessoas vivendo com a doença, o que representa $6,9 \%$ da população nacional ${ }^{4}$. A exposição prolongada à hiperglicemia leva ao desenvolvimento de complicações microvasculares; a glicólise excessiva leva ao aumento do fluxo de elétrons na cadeia de transportadores de elétrons mitocondrial com aumento da produção de espécies reativas de oxigênio, que são moléculas quimica- mente instáveis, altamente reativas que induzem dano celular 5 .

A neuropatia é uma complicação comum das diabetes tipo 1 e tipo 2. A prevalência de neuropatia é estimada em cerca de $8 \%$ em pacientes recém diagnosticados e maior que $50 \%$ em pacientes com doença de longa data ${ }^{1}$. A neuropatia sensório-motora é marcada por dor, parestesia e perda sensorial. Os mecanismos envolvidos nas diferentes sensações de dor ainda são mal compreendidos, mas há ampla evidência de que descargas anormais de neurônios somatossensoriais doentes são responsáveis ${ }^{3,5}$.

A dm 1 apresenta um grande impacto na morbimortalidade pelo desenvolvimento das complicações crônicas micro e macrovasculares4. Considerando-se a escassez de estudos na literatura sobre a prevalência das neuropatias autonômicas (na) na população brasileira com dm 1, este estudo torna-se relevante na identificação precoce desta complicação bem como na determinação de possíveis fatores clínico-laboratoriais relacionados sua presença. Neste sentido, o objetivo deste artigo e evidenciar o perfil de publicações sobre neuropatias diabéticas expressos na literatura internacional e seus impactos na saúde pública.

\section{MÉTODO}

Trata-se de uma revisão integrativa da literatura. Método que se caracteriza por reunir e sintetizar resultados de pesquisas sobre um tema, de maneira sistemática e ordenada. A pergunta de pesquisa foi definida a partir da estratégia pico, que prevê a definição do participante (p), intervenção (i), comparação (c) e desfecho/ outcomes (o).

Pretende-se responder à questão norteadora: quais fatores identificados na literatura (o), estão associados a neuropatias (i) em pessoas diabéticas (p)? Em seguida, as palavras-chave "neuropatias diabéticas", "neuralgia", "fatores de risco", "diabetes mellitus" foram definidas a partir do vocabulário dos descritores em ciências da saúde (decs), por ser uma terminologia comum à pesquisa. Estas, foram combinadas entre si, utilizando-se o operador booleano and nas bases de dados e/ou bibliotecas eletrônicas: medical literature analysis and retrieval system online (medline), da biblioteca virtual de saúde (bvs) e da scientific eletronic library online (scielo). A mesma estratégia de busca foi realizada em todas as bases de dados e/ ou bibliotecas eletrônicas. Os critérios de inclusão dos artigos para análise foram: grupo populacio- 


\section{artigo}

Robson da Silva, R.; Souza, M.V.L.; Alencar, I.F.; Inácio, A.F.L.; Ferreira da Silva, D.; Messias, I.F.; Magalhães, A.F.L.i

Neuropatias diabéticas periféricas como complicações do diabetes mellitus: estudo de revisão

\section{Quadro 1 - níveis de evidência. Rio De Janeiro, RJ, Brasil, 2021}

\begin{tabular}{|c|c|c|}
\hline NIIVEL & DEFINIÇÃo & IMPLICAçõES \\
\hline Alto & $\begin{array}{c}\text { Há forte confiança de que o verdadeiro efeito esteja } \\
\text { próximo daquele estimado }\end{array}$ & $\begin{array}{c}\text { É improvável que trabalhos adicionais irão modificar a confiança na } \\
\text { estimativa do efeito }\end{array}$ \\
\hline Moderado & Há confiança moderada no efeito estimado & $\begin{array}{c}\text { Trabalhos futuros poderão modificar a confiança na estimativa } \\
\text { de efeito, com possibilidade, inclusive, de modificar a estimativa }\end{array}$ \\
\hline Baixo & A confiança no efeito é limitada & $\begin{array}{c}\text { Trabalhos futuros provavelmente terão um impacto importante em } \\
\text { nossa confiança na estimativa de efeito }\end{array}$ \\
\hline Muito baixo & $\begin{array}{c}\text { A confiança na estimativa de efeito é muito limita- } \\
\text { da. Há importante grau de incerteza nos achados }\end{array}$ & Qualquer estimativa de efeito é incerta \\
\hline Fonte: os autores, 2021
\end{tabular}

Figura 1 - síntese dos resultados da revisão sistemática. Rio de janeiro, RJ, 2021.

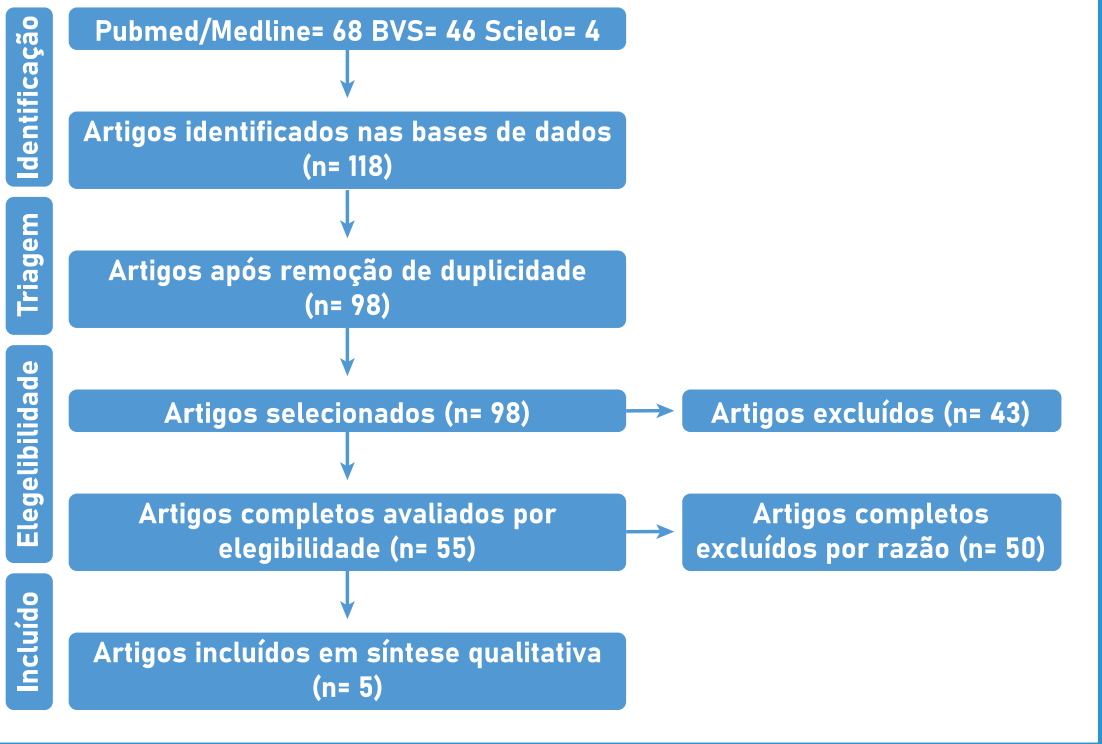

Fonte: elaborado pelos autores, 2021.

nal de pessoas diabéticas, publicados entre 2011 e 2021, disponíveis na íntegra, nos idiomas português, inglês e espanhol, que tratavam sobre a temática de neuropatias diabéticas.

Foram excluídos artigos de opinião, editoriais, outras revisões, artigos duplicados e publicações que não tratassem da temática. O período de coleta ocorreu nos meses de fevereiro a março de 2021. Para a análise dos dados, foi construído um quadro analítico que possibilitou reunir e sintetizar as informações-chave dos estudos. O instrumento de coleta reuniu as seguintes informações: título, autor(es)/ ano de publicação/país, objetivo, método, principais resultados. O nível de evi- dência identificado nos artigos analisados foi classificado de acordo com o sistema grading of recommendations assessment, development and evaluation (grade) um sistema considerado sensível para graduar a qualidade das evidências. Nesse sistema, a qualidade da evidência é descrita em quatro níveis: alto, moderado, baixo e muito baixo (quadro 1).

A evidência proveniente de ensaios clínicos randomizados começa com nível alto e a evidência proveniente de estudos observacionais, com nível baixo. Nesta revisão, a partir da classificação adotada (sistema grade) para avaliar a qualidade das evidências, considerou-se o risco de viés dos ensaios clínicos randomizados das tecnologias de produto em relação às limitações metodológicas referentes ao delineamento ou à execução dos estudos individuais. As evidências dos ensaios clínicos randomizados podem ser rebaixadas por ausência de sigilo da alocação, ausência de cegamento, seguimento incompleto, relato seletivo de desfechos e outras limitações, como interrupção precoce do estudo por benefício e informações insuficientes para avaliar se há importante risco de viés. Para cada um desses domínios é avaliado o risco de viés, sendo classificado como alto risco, incerto e baixo risco de viés.

\section{RESULTADO}

Identificou-se, um total de 118 estudos nas referidas bases de dados, conforme ilustrado na (figura 1), a qual seguiu as recomendações prisma. Para descrever o processo de busca na literatura. Desses, foram excluídos 20 artigos duplicados, restando 98 artigos únicos. Em seguida, realizou-se a leitura dos títulos e resumos observando-se os critérios de inclusão e exclusão. Como resultado desse processo, 43 artigos foram excluídos e outros 55 artigos se adequaram aos critérios de elegibilidade. Iniciou-se, então, a leitura integral e em profundidade desses estudos por dois pesquisadores, independentemente.

Eventuais discordâncias entre os avaliadores, surgidas durante esse estágio, foram trabalhadas e resolvidas mediante consenso, o que resultou em uma amostra final de 5 artigos. Os artigos incluídos nesta síntese (tabela 1) foram desenvolvidos em seis diferentes países: brasil $(\mathrm{n}=1)$, esta- 
dos unidos $(n=2)$, taiwan $(n=1)$, áfrica $(\mathrm{n}=1)$ quanto ao método, a maioria dos pesquisadores utilizou a abordagem qualitativa e quantitativa $(n=5)$ para descrever e analisar, em profundidade, as diferentes dimensões em que ocorre a neuropatia diabética.

\section{DISCUSSÃO}

Várias síndromes bastante distintas de neuropatia diabética foram delineadas ${ }^{6}$. A mais comum, como observado, é uma polineuropatia distal, simétrica, principalmente sensorial, que afeta os pés e as pernas de maneira crônica e lentamente progressiva ${ }^{7}$. Os outros são os seguintes: oftalmoplegia aguda que afeta o terceiro, e menos frequentemente o sexto nervo craniano de um lado; mononeuropatia aguda de membros ou tronco incluindo uma radiculopatia toracolombar dolorosa8; uma neuropatia múltipla

Tabela 1 - síntese dos estudos publicados, Rio De Janeiro, RJ, 2021.

\section{TÍTULOS}

Diabetic polyneuropathy with/out neuropathic pain in mali: a cross-sectional study in two reference diabetes treatment centers in bamako (mali)

Prevalence and biochemical risk factors of diabetic peripheral neuropathy with or without neuropathic pain in taiwanese adults with type 2 diabetes mellitus

Fatores associados a alteração da percepção sensorial tátil nos pés de pacientes com diabetes mellitus $\mathrm{tt}$ - facrors associated with altered tactile sensory perception in the feet of patients with diabetes mellitus

Treatment-induced neuropathy of diabetes: an acute, iatrogenic complication of diabetes

Sensory phenotype and risk factors for painful diabetic neuropathy: a cross-sectional observational study
Maiga, y. Et al. Africa (2020)

AUTOR(ES), PAÍS/ ANO

OBJETIVO

MÉTODO

Determinar a prevalência da polineuropatia diabética.

Estudo transversal descritivo

Investigar a prevalência e os fatores de

Pai, y. Wei, taiwan (2018) Noronha, j. etiológicos da alteA. F. Brasil ração da percepção (2019)

Gibbons, c., freeman, $r$. Estados unidos (2015)

Raputova, j. Et al. Estados unidos (2016) risco para neuropatia periférica diabética com ou sem dor neuropática em

taiwaneses.

Analisar sinais, sintomas e fatores
Identificar a prevalência e os fatores de risco deste transtorno não são conhecidos.

\section{Estudo observa- cional trans- versal}

Estudo transversal quantitativa sensoril tátil em pamellitus

Qualitativo transversal

Estudo transversal observacional
Caracterizar os fenótipos sensoriais de pacientes com neuropatia diabética dolorosa e indolor e avaliar os parâmetros demográficos.

\section{RESULTADOS}

A prevalência de polineuropatia diabética baseada em serviços de saúde com ou sem dor neuropática foi alta em nossa coorte $69,8 \%$

(176/252).

0 risco de neuropatia diabética periférica com dor neuropática deve ser considerado para pessoas com idade avançada, hemoglobina glicada elevada, colesterol de lipoproteína de alta densidade baixa

Constatou-se uma alta prevalência de alteração na percepção sensorial tátil entre os diabéticos

A neuropatia do diabetes induzida por tratamento é um distúrbio iatrogênico subestimado, associado a complicações microvasculares difusas. A mudança glicêmica rápida em pacientes com diabetes não controlada aumenta o risco dessa complicação.

NÍVEL DE EVIDÊNCIA

Baixo

Baixo

Moderado

Moderado

A dor neuropática foi positivamente correlacionada com a gravidade da neuropatia e hipossensiAlto 
dolorosa aguda ou subaguda, assimétrica, predominantemente motora que afeta as raízes lombares superiores e os músculos proximais da perna (amiotrofia diabética); uma fraqueza motora proximal geralmente sem dor e com perda sensorial variável ${ }^{9}$, seguindo um curso subagudo ou crônico, e uma neuropatia autonômica envolvendo intestino, bexiga, sudorese e reflexos circulatórios ${ }^{10}$.

Essas formas de neuropatia frequentemente coexistem ou se sobrepõem, particularmente os tipos autonômicos e distais simétricos e as neuropatias proximais subagudas $^{10,11}$. A polineuropatia diabética sensorial distal é a apresentação mais comum de neuropatia no diabetes, e até $50 \%$ dos pacientes podem apresentar sintomas mais frequentemente como dor em queimação, sensações elétricas ou pontadas, parestesia, hiperestesia e dor profunda ${ }^{12}$. Esses sintomas geralmente pioram à noite e perturbam o sono. Juntamente com os sintomas dolorosos durante o dia, isso geralmente leva a uma redução na capacidade do indivíduo de realizar as atividades diárias ${ }^{13}$

A fraqueza muscular é geralmente leve, mas em alguns pacientes uma neuropatia sensorial distal é combinada com uma fraqueza proximal e atrofia ${ }^{7,14}$. A neuropatia craniana em pacientes diabéticos envolve mais comumente o nervo oculomotor, seguido pelos nervos troclear e facial em ordem de freqüência ${ }^{15}$. A paralisia do terceiro nervo com preservação pupilar é a marca registrada da paralisia oculomotora diabética e é atribuída ao infarto do nervo. Raramente um nervo da extremidade superior é afetado ${ }^{16}$. Nestes casos, o encarceramento do nervo parece ser mais comum do que o infarto do nervo ${ }^{17}$. As mononeuropatias frequentemente surgem durante os períodos de transição na doença diabética, por exemplo, após um episódio de hiper ou hipoglicemia, quando o tratamento com insulina é iniciado ou ajustado, ou quando ocorre uma rápida perda de peso ${ }^{18}$. Mononeuropatias múltiplas e radiculopatias diabéticas uma síndrome de neuropatias múltiplas unilaterais ou assimétricas dolorosas tende a ocorrer em pacientes mais velhos com diabetes relativamente leve ou mesmo não reconhecido ${ }^{4,5,7}$.
A neuropatia diabética radiculoplexo lombossacral ocorre em aproximadamente $1 \%$ dos pacientes diabéticos e é provavelmente a forma de neuropatia diabética que causa a maior morbidade ${ }^{2,5}$. Tem sido conhecido por diferentes nomes, incluindo amiotrofia diabética, síndrome de bruns-garland, mononeurite múltipla diabética, polirradiculopatia diabética, neuropatia diabética proximal e outros ${ }^{2,5,6,19}$. As radiculopatias torácicas diabéticas são uma complicação rara, mas importante, do diabetes mellitus ${ }^{3}$. Estes tipicamente se manifestam com dor intensa e disestesia ao longo do tronco, tórax ou parede abdominal, e muitas vezes solicitam exames extensivos para patologia torácica ou abdominal subjacente ${ }^{4,8}$. Eles podem ser simétricos e podem envolver vários dermátomos. Embora a neuropatia radiculoplexo lombossacral diabética seja um ramo muito mais familiar do espectro da neuropatia radiculoplexo, o segmento cervical também pode estar envolvido, mas isso é muito raro ${ }^{17}$.

A neuropatia autonômica diabética é um distúrbio generalizado das fibras autonômi-

No diabetes, a nac

é o resultado de interações complexas entre o grau de controle glicêmico, duração da doença, desgaste neuronal relacionado à idade

e a pressão arterial sistólica e diastólica cas colinérgicas, adrenérgicas e peptidérgicas no contexto do diabetes sem outras causas. É caracterizada por uma forma subclínica detectável apenas por exames, e por uma forma clínica com presença de sinais e sintomas ${ }^{14,15}$. Neuropatia autonômica cardiovascular (nac) é definida como o comprometimento do controle autonômico do sistema cardiovascular ${ }^{20}$. No diabetes, a nac é o resultado de interações complexas entre o grau de controle glicêmico, duração da doença, desgaste neuronal relacionado à idade e a pressão arterial sistólica e diastólica ${ }^{19}$.

A hiperglicemia desempenha um papel fundamental na ativação de várias vias bioquímicas relacionadas ao estado metabólico e/ou redox da célula, que, em conjunto com a perfusão nervosa prejudicada, contribui para o desenvolvimento e progressão das neuropatias diabéticas ${ }^{1,2}$. Como a neuropatia é vista primeiro nas fibras mais longas, as primeiras manifestações de neuropatia autonômica no diabetes tendem a estar associadas à desnervação parassimpática, com o consequente aumento precoce do tônus simpático $^{19}$. Os sintomas clínicos de disfunção autonômica podem não aparecer até muito depois do início do diabetes ${ }^{2}$. No entanto, a nac subclínica pode ser detectada dentro de 1 ano do diagnóstico no diabetes tipo 2 e dentro de 2 anos do diagnóstico no diabetes tipo $1^{16}$. Os sinais desta doença são diminuição da variabilidade da frequência cardíaca, taquicardia em repouso, intolerância ao exercício, regulação anormal da pressão arterial e hipotensão ortostática ${ }^{12}$. Nac está significativamente associado à mortalidade, como isquemia miocárdica silenciosa, doença arterial coronariana, acidente vascular cerebral, progressão da nefropatia diabética e morbidade perioperatória?.

Assim, a avaliação nac pode ser usada para estratificação de risco cardiovascular em pacientes com e sem doença cardiovascular estabelecida ${ }^{6,19}$. Neuropatia autonômica gastrointestinal as funções motoras gastrointestinais, sensoriais e secretoras são moduladas pela interação dos sistemas nervoso autônomo (simpático e parassimpático) e entérico com ritmicidade subjacente gerada pelas células intersticiais de cajal localizadas dentro do músculo liso8. A avaliação da fun- 
ção autonômica gastrointestinal é difícil em humanos, e o diagnóstico de neuropatia autonômica gastrointestinal costuma ser de exclusão 5 . O diabetes está associado a sintomas gastrointestinais, como náusea, distensão abdominal, dor abdominal, diarreia, constipação e esvaziamento gástrico retardado com concomitante redução da absorção de drogas orais, controle glicêmico deficiente, desnutrição, regulação pós-prandial anormal da pressão arterial, baixa qualidade de vida e alta taxa de hospitalização9,11.

Até 75\% dos pacientes com diabetes podem apresentar esses sintomas. A disfunção erétil (de) afeta 30-40\% dos homens diabéticos e ocorre como resultado de disfunção endotelial e neuropatia autonômica ${ }^{6}$. A estreita associação com a disfunção endotelial significa que a de é um marcador precoce de risco cardiovascular' ${ }^{9}$ Embora existam alguns tratamentos eficazes para a de, o manejo dessa condição às vezes é extremamente difícil ${ }^{18}$. É recomendável fazer parte de um serviço de atendimento ao diabetes ${ }^{2}$. O tratamento eficaz da disfunção erétil requer o reconhecimento da condição e de suas comorbidades associadas, incluindo disfunção endotelial e disfunção da bexiga diabética? .

As estimativas da prevalência de disfunção da bexiga são 43-87\% dos pacientes diabéticos tipo 1 e $25 \%$ dos pacientes diabéticos tipo ${ }^{29}$. A causa da disfunção da bexiga no diabetes mellitus é principalmente a neuropatia periférica e autonômica ${ }^{10}$. Estudos em animais e humanos revelaram que a cistopatia diabética se desenvolve como resultado da polineuropatia, que afeta predominantemente as fibras nervosas sensoriais e autonômicas. ${ }^{6}$ Os pacientes frequentemente permanecem assintomáticos nos estágios iniciais, apesar da anormalidade vesical demonstrável. Sensação da bexiga prejudicada geralmente é a primeira manifestação de envolvimento do trato urinário inferior ${ }^{9}$. Os reflexos de micção são retardados devido à diminuição da sensação da bexiga com aumentos na capacidade da bexiga e retenção urinária que geralmente ocorrem de forma assintomática ${ }^{5,19}$.

Os pacientes frequentemente desconhecem a disfunção da bexiga até que tenham uma infecção do trato urinário secundária ao aumento do volume residual de urina. Os sintomas comuns são esforço, hesitação e fraqueza do fluxo ${ }^{3,4}$. A cistopatia diabética é caracterizada por sensação prejudicada de plenitude vesical, o que leva a bexiga a ficar hiperestendida, redução da contratilidade da bexiga, aumento da urina residual e urofluxo prejudicado $^{8,19}$. A neuropatia autonômica diabética inicialmente resulta em uma perda da sudorese termorreguladora em uma distribuição que pode se estender às partes superiores dos membros e abdome anterior ${ }^{14}$.

Em última análise, esse processo resulta em anidrose global que geralmente acompanha uma neuropatia autonômica grave. A neuropatia autonômica diabética também pode causar hiperidrose ${ }^{20}$. A sudorese excessiva pode ocorrer como um processo compensatório, envolvendo regiões proximais, como a cabeça e o tronco, que são poupadas em uma neuropatia agonizante ${ }^{21}$. A sudorese gustativa, produção anormal de suor que aparece na face, cabeça, pescoço, ombros e tórax após o consumo de alimentos não condimentados, ocorre em casos ocasionais ${ }^{22}$. Uma limitação desse estudo foi a escassez de pesquisas relacionadas ao tema, mesmo sendo um assunto que deveria ser tratado com máxima importância e urgência, pois se trata de um problema que afeta uma grande parcela da população. É recomendado que mais pesquisas de campo sejam realizadas para que tenhamos uma maior dimensão do problema e assim traçar estratégias para mitigar os danos e beneficiar a comunidade.

\section{CONCLUSÃO}

As neuropatias sensório-motoras e cardiovasculares são comuns em pacientes diabéticos. Além do controle glicêmico estrito, nenhuma outra abordagem terapêutica existe na prevenção desse fenômeno. As razóes pelas quais apenas alguns pacientes com lesões nervosas desenvolvem dor neuropática ainda são desconhecidas. Fatores de risco como idade, sexo, tabagismo são predispostos a doença. A intensidade da dor antes e depois da lesão e características emocionais e cognitivas indicam que existem vários fatores além da própria lesão nervosa que contribuem para a manifestação da dor crônica. O diagnóstico e o tratamento sintomático são essenciais para esses pacientes, pois as neuropatias sensório-motoras dolorosas estão associadas à baixa qualidade de vida e as neuropatias autonômicas estão associadas ao aumento da mortalidade cardiovascular. A terapia intensiva do diabetes, a redução do risco cardiovascular multifatorial intensivo e a intervenção no estilo de vida são recomendados em pacientes com nac. O tratamento sintomático dos sintomas sensoriais inclui tcas, snris, gabapentina, pregabalina e opióides.

\section{REFERÊNCIAS}

1. Raputova j, srotova i, vlckova e, sommer c, üçeyler $n$, birklein $f$, et al. Sensory phenotype and risk factors for painful diabetic neuropathy: a cross-sectional observational study. Pain. 2017 dec;158(12):2340-53.

2. Carvalho acv, domingueti cp. Papel das citocinas inflamatórias na nefropatia diabética tt - role of inflammatory cytokines in diabetic nephropathy. Rev soc bras clín méd [internet]. 2016;14(3):177-82. Available from: http://fi-admin.bvsalud.org/ document/view/7n7vc
3. Oliveira $\mid z$, bavaresco ss, cumplido mgr, pillatt ap, marchi acb, leguisamo cp. Grau de risco para desenvolvimento de úlceras nos pés de pacientes diabéticos de meia idade e idosos tt - degree of risk for developing foot ulcers of diabetic middle-aged and elderly. Fisioter bras [internet]. 2013;14(6):459-63. Available from: https://pesquisa.bvsalud.org/portal/resource/pt/lil-789861

4. Scain sf, franzen e, hirakata vn. Effects of nursing care on patients in an educational program for prevention of diabetic foot. $\mathrm{Tt}$ - riscos associados à mortalidade em pacientes atendidos em 


\section{REFERÊNCIAS}

um programa de prevenção do pé diabético. Rev gauch enferm [internet]. 2018;39:e20170230-e20170230. Available from: http://www.scielo.br/scielo.php?script=sci_arttext\&amp

5. Vinik ai, tuchman m, safirstein b, corder c, kirby l, wilks k, et al. Lamotrigine for treatment of pain associated with diabetic neuropathy: results of two randomized, double-blind, placebo-controlled studies. Pain. 2007 mar;128(1-2):169-79.

6. Palma fh, antigual du, martínez sf, monrroy ma, gajardo re. Static balance in patients presenting diabetes mellitus type 2 with and without diabetic polyneuropathy tt - equilíbrio estático em pacientes com diabetes melito tipo 2 com ou sem polineuropatia diabética. Arq bras endocrinol metab [internet]. 2013;57(9):722-6. Available from: http://www.scielo.br/scielo. php?script=sci_arttext\&amp

7. Brinati Im, diogo nas, moreira tr, mendonça ét, amaro mof. Prevalência e fatores associados à neuropatia periférica em indivíduos com diabetes mellitus tt - prevalencia y factores asociados con la neuropatía periférica en individuos con diabetes mellitus tt - prevalence and factors associated with peripheral ne. Rev pesqui (univ fed estado rio j, online) [internet]. 2017;9(2):347-55. Available from: http://www.seer.unirio.br/ index.php/cuidadofundamental/article/view/4476/pdf

8. Gibbons ch, freeman r. Treatment-induced neuropathy of diabetes: an acute, iatrogenic complication of diabetes. Brain. 2015 jan;138(pt 1):43-52.

9. Barrile sr, ribeiro aa, costa apr da, viana aa, conti mhs de, martinelli b. Comprometimento sensório-motor dos membros inferiores em diabéticos do tipo 2 tt - sensitive-motor alteration of the lower limbs in diabetics type 2. Fisioter mov [internet]. 2013;26(3):537-48. Available from: http://www.scielo.br/scielo. php?script=sci_arttext\&amp

10. Vasco bb, ferraz c, alves gv, cagnin gt, mizuno tm, stuchi-perez eg. Elaboração de protocolo de investigação de neuropatia periférica em pacientes diabéticos tt - elaboration of a protocol for peripheral neuropathy investigation in diabetic patients tt - elaboración de protocolo de investigación de neuropatía periférica . Cuid enferm [internet]. 2019;13(1):22-6. Available from: http://www.webfipa.net/facfipa/ner/sumarios/ cuidarte/2019v1/22.pdf

11. Pai $y-w$, lin $c-h$, lee $i-t$, chang $m-h$. Prevalence and biochemical risk factors of diabetic peripheral neuropathy with or without neuropathic pain in taiwanese adults with type 2 diabetes mellitus. Diabetes metab syndr. 2018;12(2):111-6.

12. Deli g, bosnyak e, pusch g, komoly s, feher g. Diabetic neuropathies: diagnosis and management. Neuroendocrinology. 2013;98(4):267-80.

13. Noronha jaf. Fatores associados a alteração da percepção sensorial tátil nos pés de pacientes com diabetes mellitus tt facrors associated with altered tactile sensory perception in the feet of patients with diabetes mellitus [internet]. 2019. P. 177. Available from: http://hdl.handle.net/1843/enfc-bcehsv

14. Figueiredo éoc de, barros fo, santos ef dos, pimentel ts, góis cfl, otero Im. Avaliação do grau de risco para pé diabético em indivíduos com diabetes mellitus tipo 2 tt - risk level evaluation for diabetic foot in individuals with type 2 diabetes mellitus. Rev enferm ufpe line [internet]. 2017;11(supl.11):4692-9. Available from: https://periodicos.ufpe.br/revistas/revistaenfermagem/ article/view/231211/25218

15. Hébert hl, veluchamy a, torrance n, smith bh. Risk factors for neuropathic pain in diabetes mellitus. Pain. 2017 apr;158(4):560-8.

16. Silva jv da, sousa-muñoz rl de, figueiredo âs de, melo jfg de, fernandes bm. Fatores de risco para perda de sensibilidade plantar em diabéticos: estudo caso-controle em ambulatório de endocrinologia tt - risk factors for loss of plantar sensitivity in diabetics: a case-control study in endocrinology outpatient clinic. Rev bras ciênc saúde [internet]. 2013;17(2):113-20. Available from: http://periodicos.ufpb.br/ojs/index.php/rbcs/article/ download/15028/9703

17. Pedras $s$, carvalho $r$, pereira $m$ da g. Sociodemographic and clinical characteristics of patients with diabetic foot ulcer. Rev assoc med bras. 2016 apr;62(2):171-8.

18. Maiga $y$, diallo $s$, konipo fdn, sangho o, sangaré $m$, diallo sh, et al. Diabetic polyneuropathy with/out neuropathic pain in mali: a cross-sectional study in two reference diabetes treatment centers in bamako (mali), western africa. Plos one. 2020;15(11):e0241387.

19. Braga dc, bortolini sm, rozetti ig, zarpellon k, nascimento jc, neris je. Avaliação de neuropatia e complicações vasculares em pacientes com diabetes mellitus em um município rural de santa catarina tt - evaluation of neuropathy and vascular complications in patients with diabetes mellitus in a rural town of santa catarina. Rev amrigs [internet]. 2015;59(2):78-83. Available from: http://www.amrigs.org.br/revista/59-02/02_1453_revista amrigs.pdf

20. Mello $r$ da $f$ de a. Ficha de avaliação clínica de membros inferiores para prevenção do pé diabético tt - lower limb clinical assessment form for diabetic foot prevention [internet]. 2016. P. 105. Available from: http://fi-admin.bvsalud.org/document/ view/m64wb

21. Silva rr da, silva la da, silva mvg da, neves $\mathrm{mp}$ das, silva $\mathrm{mm}$ dos s da, francisco mtr, Marta cb. Os impactos do chemsex na saúde pública mundial: um estudo sobre uma perigosa prática sexual entre homens. Saudcoletiv (barueri) [internet]. $29^{\circ}$ de outubro de 2020 [citado $19^{\circ}$ de maio de 2021];9(51):1920-5. Disponível em: http://revistas.mpmcomunicacao.com.br/index.php/ saudecoletiva/article/view/177

22.De souza francisco barbosa i, de fátima de sousa mendonça $c$, andrade da silva l, Preissler das neves m, da silva rr, ferracini de alencar ícaro, da silva de araújo m. Produções acadêmicas sobre a assistência destinada às pessoas com problemas relacionados ao abuso de álcool. Um estudo de revisão. Saudcoletiv (barueri) [internet]. $6^{\circ}$ de abril de 2021 [citado $19^{\circ}$ de maio de 2021];11(63):5424-35. Disponivel em: http://revistas.mpmcomunicacao.com.br/index.php/saudecoletiva/article/view/1450 